\title{
Low serum bilirubin levels associated with subclinical atherosclerosis in patients with obstructive sleep apnea
}

\author{
HAKAN DUMAN $^{1, *}$, SONGÜL ÖZYURT ${ }^{2}$
}

\author{
${ }^{1}$ Faculty of Medicine, Department of Cardiology, Recep Tayyip Erdoğan University, Rize, Turkey \\ ${ }^{2}$ Faculty of Medicine, Department of Chest Diseases, Recep Tayyip Erdoğan University, Rize, Turkey \\ *Corresponding author: Hakan Duman, MD; Faculty of Medicine, Department of Cardiology, Recep Tayyip Erdoğan University, 53100 Rize, \\ Turkey; Phone: +90 46421304 91; Fax: +90 46421703 64; E-mail: drhakanduman@hotmail.com
}

(Received: June 7, 2018; Revised manuscript received: July 3, 2018; Accepted: July 9, 2018)

\begin{abstract}
Background: Obstructive sleep apnea (OSA) is a chronic, progressive disease that can cause cardiovascular complications and atherosclerosis. We evaluated the relationship between serum bilirubin levels and carotid intima-media thickness (cIMT), as a surrogate marker of subclinical atherosclerosis, in patients with OSA. Methods: We enrolled 84 consecutive patients with OSA. The patients underwent ultrasonography, polysomnography, and echocardiography. Blood samples were obtained from all of the subjects, which were used for biochemical comparisons. The patients were divided into groups according to cIMT values $(<0.9$ vs. $>0.9 \mathrm{~mm})$. Results: The patient population consisted of 84 OSA patients (mean age: $54.2+8.0$ years, $62.9 \%$ male). The OSA patients with cIMT $\geq 0.9$ had significantly elevated high-sensitive C-reactive protein (hsCRP), Oxygen desaturation index, apnea-hypopnea index (AHI), and significantly lower total and indirect bilirubin levels. Multivariate regression analyses revealed that total bilirubin, hsCRP, and AHI were the independent predictors of subclinical atherosclerosis. There was a negative correlation between total bilirubin and cIMT. Conclusions: This study showed that high hsCRP and low serum total bilirubin levels could be paving the way for the development of subclinical atherosclerosis. Simple measures such as total bilirubin may provide predictive information regarding the risk of cardiovascular disease in patients with OSA.
\end{abstract}

Keywords: bilirubin, obstructive sleep apnea, carotid intima-media thickness, antioxidant, subclinical atherosclerosis

\section{Introduction}

The recondite effects of many products within the human metabolism are being defined in this day and age. One of the end products of heme metabolism, bilirubin has long been recognized as a toxic waste with a toxic effect on the nervous system. However, recent evidence shows that bilirubin could have many benefits including antioxidant and anti-inflammatory effects [1]. Similar to lipid and lipoprotein, serum bilirubin levels were discovered to be a risk factor predictive of coronary heart disease [2]. In the Framingham offspring study, higher serum bilirubin concentrations in men were detected to have a relationship with a lower risk of myocardial infarction, coronary death, and any cardiovascular events; however, no clear pattern was discovered in women [3]. The meta-analysis of 11 studies was an indication that there was an inverse relationship between increased bilirubin concentrations and atherosclerosis severity in men. There was a $6.5 \%$ decrease in cardiovascular events with every $1 \mathrm{~mol} / \mathrm{L}$ increase in bilirubin in this study [4]. Obstructive sleep apnea (OSA) is a complex syndrome characterized by oxygen desaturation and sleep disorder as well as upper airway obstruction experienced recurrently during sleep. Apneic attacks related to low oxygen desaturation, stimulations due to upper airway collapse, and recurrent activation attacks of the sympathetic nervous system could cause negative intrathoracic pressure, abnormal, humoral, neural metabolic, and cardiovascular responses as well as thrombotic and inflammatory responses that contribute to mortality [5]. Atherosclerosis is an inflammatory process that starts during childhood and develops stages in all phases of life [6].

This is an open-access article distributed under the terms of the Creative Commons Attribution-NonCommercial 4.0 International License, which permits unrestricted use, distribution, and reproduction in any medium for non-commercial purposes, provided the original author and source are credited, a link to the CC License is provided, and changes - if any - are indicated. 
cIMT is frequently used as a significant indicator in terms of subclinical or early diagnosis of atherosclerosis in epidemiological and interventional studies. Several studies regarding the association of cIMT with undesired cardiovascular events have evaluated the association of cIMT with cardiovascular disease incidence, including both coronary heart disease and stroke, to determine whether this measurement can also predict future cardiovascular events $[7,8]$. Oxidative stress is a pivotal feature of the atherogenesis. Furthermore, oxidative stress in OSA correlates with surrogate markers of endothelial function, intimamedia thickness, and high blood pressure. Bilirubin is an endogenous antioxidant in human vascular endothelial cells. The aim of this study is to analyze the relationship between the intima-media thickness in the carotid system and the serum bilirubin levels in OSA patients.

\section{Materials and Methods}

\section{Study population}

Newly diagnosed as having polysomnography, 84 consecutive patients were enrolled into the study. After the exclusion based on the exclusion criteria, the patients were categorized based on age and sex, and 84 people were divided into two groups: $<0.9 \mathrm{~mm}(n=41) ; \geq 0.9 \mathrm{~mm}$ $(n=43)$ based on their carotid intima-media measurements [9]. The exclusion criteria were chronic obstructive pulmonary disease, secondary hypertension reasons, diabetes mellitus, congestive heart failure, a history of coronary artery disease (CAD), cerebrovascular disease, carotid artery stenosis greater than $50 \%$, peripheral artery disease, antidiabetic and lipid-lowering agents, for any reason of renal or liver failure, thyroid disorders, malignancy, systemic infections during the study or within 2 weeks before the study, and chronic systemic inflammatory diseases. The study protocol was approved by local ethics committee, and all the patients provided written informed consent to participate in the study. All patients underwent physical examination and blood pressure measurement through brachial artery with a sphygmomanometer after a 5-min rest. Body mass index (BMI) was calculated by dividing the body weight $(\mathrm{kg})$ by the height $(\mathrm{m})$ squared.

\section{Polysomnography}

All patients were involved in an extensive sleep study in the sleep laboratory of our hospital using a computer software (Alice Sleepware, Philips Respironics, Murrysville, PA, USA). Apnea was defined as a total cessation of airflow for $\geq 10 \mathrm{~s}$. Hypopnea was defined as $30 \%$ or more reduction in the respiratory airflow lasting for $>10 \mathrm{~s}$ accompanied by a decrease of $>4 \%$ in oxygen saturation. The average number of apneas and hypopneas per hour of sleep was calculated as the apnea-hypopnea index (AHI). Patients who experienced AHI events $\geq 5$ per hour were diagnosed as having OSA. Oxygen desaturation index (ODI), cumulative time percentage with $\mathrm{SpO}_{2} 90 \%$, lowest and average $\mathrm{SpO}_{2}$ were extracted from the oximetry data.

\section{Measurement of cIMT}

The carotid artery imaging was performed in a warm, silent, and dark room with the patient lain in asupine position, angling their neck $20^{\circ}$ ahead. The cIMT measurements were recorded by an experienced cardiologist who did not have previous knowledge of patients' laboratory and clinical characteristics. Carotid duplex measurements were completed with high-resolution ultrasonography GE Vivid 3 (GE Healthcare, Milwaukee, USA), $8 \mathrm{MHz}$ linear array transducers device. The measurements were taken from three points: right and left common carotid arteries and bifurcation and internal carotid artery for the first $2 \mathrm{~cm}$, and the cIMT measurements were completed evaluating only back (distant) wall. The cIMT measurements were taken longitudinally in the B-mode examination from the distance defined as between vessel lumen echogenicity and media/adventitia echogenicity. The mean cIMT was calculated based on the average of three measurements from both carotid arteries.

\section{Ehocardiography}

The echocardiographic examination was performed with subjects in the left lateral decubitus position using a commercially available ultrasound system with a $2.5-3.5 \mathrm{MHz}$ transducer (ie33, Phillips Medical System, Bothell, WA, USA). Left ventricular ejection fraction (LVEF) was estimated using Simpson's biplane method. The parameters necessary for calculation of myocardial performance index (MPI) were obtained by pulsed Doppler method in the apical four-chamber view. Right ventricle MPI calculated using (isovolumic contraction time + isovolumic relaxation time/right ventricular ejection time) formula. Tricuspid annular plane systolic excursion represents the distance of systolic excursion of the right ventricular annular plane toward the apex and is measured as the maximum apical excursion of the lateral tricuspid annular plane.

\section{Laboratory analysis}

The blood samples were collected from the patients' antecubital veins after at least $8 \mathrm{~h}$ of fasting. Fasting blood glucose, total and indirect bilirubin, creatinine, total cholesterol, triglycerides, and high-density lipoprotein cholesterol concentrations were measured routinely using an Abbott Diagnostics C8000i (Abbott, Germany) 
autoanalyzer in the biochemistry laboratory of our hospital with commercial kits. The serum high-sensitive Creactive protein (hsCRP) levels were measured using the nephalometric method in (UniCelDxC 800 System, Beckman Coulter Inc., USA).

\section{Statistical analysis}

The findings were presented in mean \pm standard deviation or in frequency and percentage. The Kolmogorov-Smirnov test was used to verify the normality of the distribution of the continuous variables. The clinical data between the two groups were statistically analyzed with the unpaired $t$-test for parametric and Mann-Whitney $U$ test for the nonparametric data. The statistical analysis was completed using PASW 18 (SPSS/IBM, Chicago, IL, USA), and the significant rate was considered 0.05 (two-way). In order to determine the independent predictors of cIMT 0.9 , multivariate logistic regression analyses were performed including parameters that were found to be significant $(p<0.05)$ in the univariate analysis. Odds ratios (ORs) and $95 \%$ confidence interval $(\mathrm{CI})$ were presented.

\section{Results}

The patient population consisted of 84 OSA patients (mean age: $54.2+8.0$ years, $62.9 \%$ male). There was no significant difference between the two groups in terms of basic clinical and risk factors. Both groups had similar BMI, glomerular filtration rate, lipid parameters, blood pressure, aspartate transaminase, alanine aminotransferase, and glucose levels $(p>0.05)$ (Table I).

Table I Baseline clinical and laboratory characteristics according to cIMT

\begin{tabular}{|c|c|c|c|}
\hline Variables & $\operatorname{cIMT}<0.9 \mathrm{~mm}(n=41)$ & $\operatorname{cIMT} \geq 0.9 \mathrm{~mm}(n=43)$ & $p$ \\
\hline Age (years) & $50 \pm 8.2$ & $49 \pm 6.8$ & 0.49 \\
\hline Hypertension $[n(\%)]$ & $20(48.8)$ & $27(62.8)$ & 0.19 \\
\hline Sex $[$ male, $n(\%)]$ & $23(56.1)$ & $26(60.5)$ & 0.68 \\
\hline Smoking $[n(\%)]$ & $7(17.1)$ & $10(23.3)$ & 0.48 \\
\hline Alcohol consumption $[n(\%)]$ & $0(0)$ & $1(97.7)$ & 0.32 \\
\hline Dyslipidemia $[n(\%)]$ & $14(34.1)$ & $17(39.5)$ & 0.60 \\
\hline $\operatorname{BMI}\left(\mathrm{kg} / \mathrm{m}^{2}\right)$ & $26.7 \pm 3.9$ & $27.4 \pm 4.2$ & 0.46 \\
\hline $\mathrm{SBP}(\mathrm{mmHg})$ & $130.8 \pm 15.7$ & $130.7 \pm 12.7$ & 0.08 \\
\hline $\mathrm{DBP}(\mathrm{mmHg})$ & $74.9 \pm 12$ & $76.1 \pm 10.5$ & 0.61 \\
\hline Hemoglobin $(\mathrm{g} / \mathrm{dl})$ & $14(11-16)$ & $14(12-17)$ & 0.13 \\
\hline White blood cell count $(\times 109 / \mathrm{L})$ & $8.11 \pm 1.1$ & $8.46 \pm 1.79$ & 0.28 \\
\hline hsCRP $(\mathrm{mg} / \mathrm{L})$ & $0.9(0.4-2.7)$ & $1.4(0.42-2.9)$ & 0.02 \\
\hline $\operatorname{GFR}\left(\mathrm{ml} / \mathrm{min} / 1.73 \mathrm{~m}^{2}\right)$ & $86.4 \pm 11.8$ & $82.5 \pm 12.5$ & 0.13 \\
\hline LDL cholesterol $(\mathrm{mg} / \mathrm{dl})$ & $142 \pm 26.7$ & $140 \pm 36.4$ & 0.85 \\
\hline HDL cholesterol $(\mathrm{g} / \mathrm{dl})$ & $34.2 \pm 8.1$ & $33.7 \pm 8.2$ & 0.80 \\
\hline Triglyceride $(\mathrm{mg} / \mathrm{dl})$ & $146(117-276)$ & $143(117-341)$ & 0.43 \\
\hline $\operatorname{AST}(\mathrm{U} / \mathrm{L})$ & $25.4 \pm 6$ & $26 \pm 6.7$ & 0.64 \\
\hline $\operatorname{ALT}(\mathrm{U} / \mathrm{L})$ & $27.5 \pm 3.6$ & $28 \pm 4.8$ & 0.62 \\
\hline Glucose $(\mathrm{mg} / \mathrm{dl})$ & $89(70-118)$ & $90(75-120)$ & 0.70 \\
\hline Total bilirubin $(\mu \mathrm{mol} / \mathrm{L})^{\mathrm{a}}$ & $12.3 \pm 4.1$ & $8 \pm 3.4$ & $<0.01$ \\
\hline Indirect bilirubin $(\mu \mathrm{mol} / \mathrm{L})$ & $8.8 \pm 3.5$ & $5.3 \pm 3.3$ & $<0.001$ \\
\hline Total bilirubin $(\mathrm{mg} / \mathrm{dl})$ & $0.72 \pm 0.24$ & $0.47 \pm 0.2$ & $<0.01$ \\
\hline Indirect bilirubin $(\mathrm{mg} / \mathrm{dl})$ & $0.51 \pm 0.2$ & $0.31 \pm 0.1$ & $<0.001$ \\
\hline AHI & $20.8 \pm 12.3$ & $28.6 \pm 15.2$ & 0.01 \\
\hline ODI & $23.2 \pm 9.4$ & $27.9 \pm 11.4$ & 0.04 \\
\hline
\end{tabular}

BMI: body mass index; cIMT: carotid intima-media thickness; DBP: diastolic blood pressure; GFR: glomerular filtration rate; hsCRP: high-sensitive C-reactive protein; HDL: high-density lipoprotein; LDL: low-density lipoprotein; SBP: systolic blood pressure; AST: aspartate aminotransferase; ALT: alanine aminotransferase; ODI: oxygen desaturation index; AHI: apnea-hypopnea index; Desaturation (\%): sleep time of $\mathrm{SpO}_{2}<90 \%$.

${ }^{a} \mathrm{SI}$ units $(\mathrm{mmol} / \mathrm{L})$ were obtained by multiplying traditional bilirubin concentrations $(\mathrm{mg} / \mathrm{dl})$ with conversion factor $(17.1)$ 
The OSA patients with cIMT $\geq 0.9$ had significantly higher hsCRP, ODI, AHI $[0.9(0.4-2.7)$ vs. 1.5 $(0.42-2.9) \mathrm{mg} / \mathrm{L}, p<0.001 ; 23.2 \pm 9.4$ vs. $27.9 \pm$ $11.4, p=0.04 ; 20.8 \pm 12.3$ vs. $30 \pm 13.8, p=0.002$, respectively] and significantly lower total and direct bilirubin levels $(12.3 \pm 4.1$ vs. $8 \pm 3.4 \mu \mathrm{mol} / \mathrm{L}, p<0.0$; $8.8 \pm 3.5$ vs. $5.3 \pm 3.3, p<0.001$, respectively). The comparison with the cIMT $\leq 0.9$ group demonstrates the relationship between total bilirubin and cIMT (Fig. 1).

There was a significant negative correlation found in the correlation analysis between total bilirubin and cIMT $(r=-0.44, p<0.01)$ (Fig. 2). Similarly, there was a significant positive correlation found in the correlation analysis between cIMT and hsCRP $(r=-0.23, p=0.03)$.

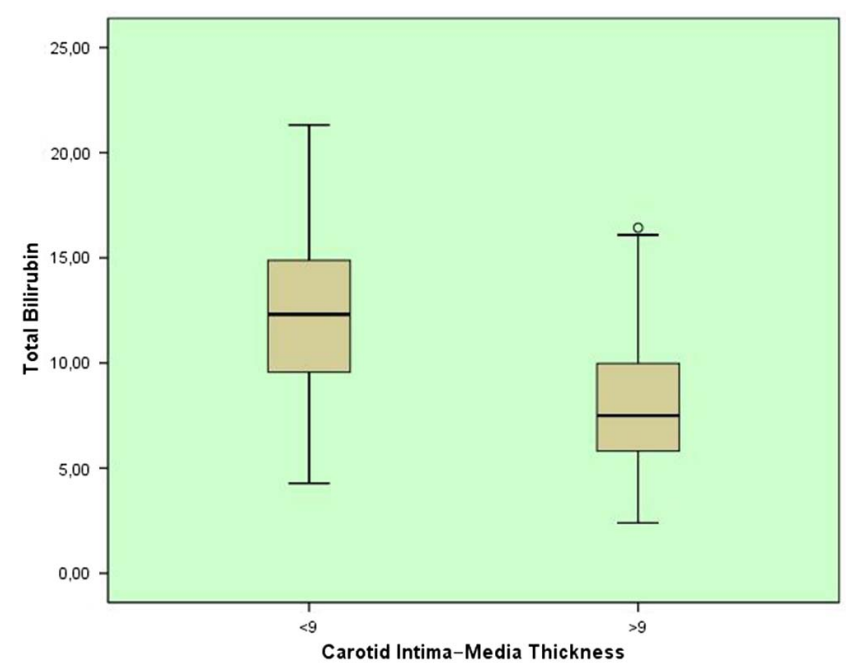

Fig. 1. Bilirubin values according to the carotid intima-media thickness

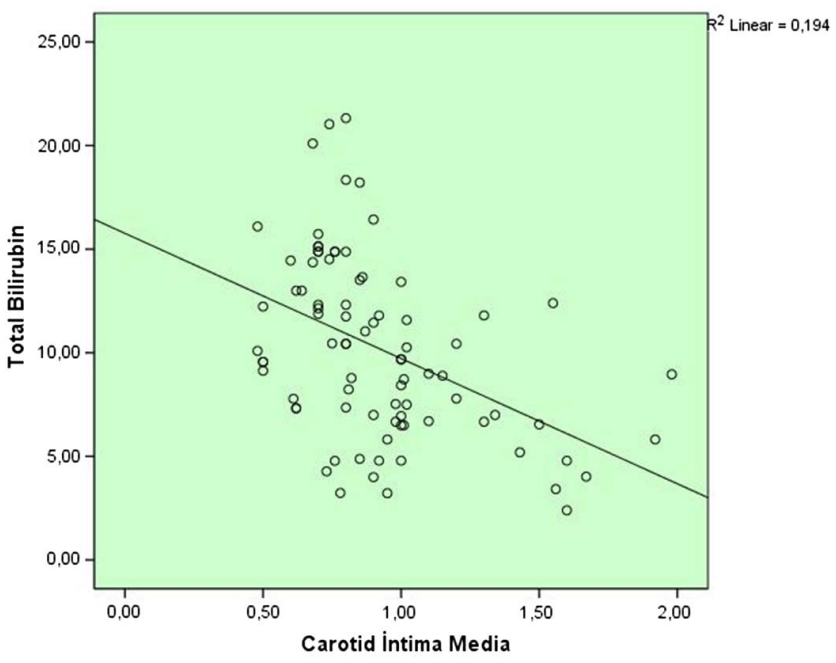

Fig. 2. Correlation graph between total bilirubin and carotid intima-media thickness
Multivariate logistic regression model was performed with hsCRP, bilirubin, ODI, and AHI independent variables. Total bilirubin (OR: 0.72, 95\% CI: 0.60-0.86, $p<0.01$ ), AHI (OR: 1.07, 95\% CI: 1.01-1.13, $p=0.01$ ), and hsCRP (OR: 2.91, 95\% CI: 1.09-7.70, $p=0.03$ ) were the independent cIMT predictors of subclinical atherosclerosis (Table II).

LVEF values obtained using echocardiographic assessment were not significantly different between the two groups. Parameters related to right ventricular function were not significantly different between the two groups (Table III).

\section{Discussion}

We have showed a study a relationship between subclinical atherosclerosis and total bilirubin levels in OSA patients. This study revealed a negative correlation between total bilirubin and cIMT. Total bilirubin, AHI, and hsCRP were found to be an independent risk factor for subclinical atherosclerosis.

OSA is the most common of the respiratory disorders during sleep characterized by repetitive obstruction episodes in the upper respiratory tract, and is usually associated with risk factors, such as obesity, hypertension, cardiovascular, and CAD [10]. Oxidative stress, vascular

Table II Multivariate logistic regression analyses, independent predictors of carotid intima-media thickness

\begin{tabular}{lcc} 
Variables & Multivariate OR [95\% CI $]$ & $p$ \\
hsCRP & $2.91[1.09-7.70]$ & 0.03 \\
Total bilirubin & $0.72[0.60-0.86]$ & 0.03 \\
Indirect bilirubin & $1.11[0.73-1.69]$ & 0.61 \\
ODI & $1.02[0.95-1.09]$ & 0.56 \\
AHI & $1.07[1.01-1.13]$ & 0.01 \\
\hline
\end{tabular}

hsCRP: high-sensitive C-reactive protein; CI: confidence interval; OR: odds ratio; ODI: oxygen desaturation index; AHI: apnea-hypopnea index

Table III $\begin{aligned} & \text { Right ventricular echocardiographic parameters of the } \\ & \text { groups }\end{aligned}$

\begin{tabular}{lllc} 
Parameters & cMIT $<0.9$ & cMIT $>0.9$ & $p$ value \\
LVEF $(\%)$ & $62.5 \pm 4.8$ & $62.0 \pm 5.2$ & 0.18 \\
RVEDD $(\mathrm{mm})$ & $38.1 \pm 3.1$ & $37.0 \pm 4.1$ & 0.41 \\
RVESD $(\mathrm{mm})$ & $19.0 \pm 3.5$ & $18.1 \pm 2.5$ & 0.21 \\
TAPSE $(\mathrm{mm})$ & $20.2 \pm 3.5$ & $19.6 \pm 4.0$ & 0.22 \\
RV MPI & $0.21 \pm 0.02$ & $0.20 \pm 0.02$ & 0.66 \\
\hline
\end{tabular}

LVEF: left ventricular ejection fraction; RVEDD: right ventricle end diastolic diameter; RVESD: right ventricle endsystolic diameter; RV MPI: right ventricular myocardial performance index; TAPSE: tricuspid annular plane systolic excursion 
endothelial dysfunction, and systemic inflammation in OSA contribute to the development of endothelial dysfunction and cardiovascular diseases [5]. Stiffening in central arteries has a number of detrimental hemodynamic consequences such as increased risk for coronary heart disease, stroke, and composite cardiovascular events [11]. Atherosclerosis is an inflammatory disease resulting from increased plasma cholesterol levels with lipid accumulation in the arterial wall in the center of lesion formation. This process includes the phagocytosis of low-density lipoprotein (LDL) cholesterol by macrophages, and is related to an increased oxidative stress, inflammatory endothelial damage $[12,13]$. Determining the preventable risk factors that affect atherosclerosis matters a great deal as this could weaken, perhaps only by a small margin, the pathological process of atherosclerosis. It has been reported that bilirubin is quite a strong antioxidant against oxidant peroxides, one of the important steps of plaque formation and atherosclerosis, and is even 30 times as strong in preventing LDL oxidation compared with a vitamin D analog [14]. Similarly, in vivo studies showed that bilirubin decreased the excessive expression of the adhesion molecules during the inflammatory process and the excessive expression of the adhesion molecules in the endothelial cells, and inhibited leukocyte endothelial adhesion molecules; all these data from the theses collectively could contribute to explaining bilirubin's protective effect on the development of atherosclerosis [15].

Previous studies reported the bilirubin's antioxidant effects and preventive side in the development of atherosclerosis [16-18]. While the mechanics of this antiatherogenic effect cannot be completely explained, it is considered that bilirubin has an antioxidant effect and it could be efficient due to this effect. Studies performed on individuals demonstrated that bilirubin has an antioxidative effect against atheromatous lesions [19, 20]. Schwertner et al. [21] reported that the decrease in the serum bilirubin levels brought along an increase in smoking and systolic blood pressure as well as in CAD. It was also reported high serum total bilirubin levels could diminish the development of early familial CAD and high levels of bilirubin could act as a protective factor [22, 23]. Another recent, retrospective, cross-sectional study by Akboğa et al. [24] found in their analysis that serum total bilirubin levels had a relationship with the severity of coronary atherosclerosis in patients with stable coronary atherosclerosis. Furthermore, Huang et al. [25] suggested the total serum bilirubin levels could be an independent predictor of long-term mortality in stable CAD (HR: $0.34,95 \% \mathrm{CI}: 0.16-0.70$ ). On the contrary, there are studies that found no significant relationship between serum bilirubin levels and atherosclerotic lesions or studies that found a U-shaped association between serum bilirubin and CAD [26, 27]. However, there can be various factors that affect atherosclerosis. Therefore, it is important to determine how each factor creates a risk independently. Our findings are seemingly in concordant with those from most of the previous studies. The relationship between the inflammatory markers of subclinical atherosclerosis in patients with OSA and cIMT was discovered in previous studies, [27] whereas this study found a statistically significant effect of serum total bilirubin levels in patients with OSA on cIMT, a potential start of the atherosclerosis process. OSA may be associated with chronic liver dysfunction due to right ventricular dysfunction. This can increase serum bilirubinin. In this study, right ventricular functions were assessed using specific echo parameters and no evidence of right ventricular dysfunction was detected. This may be due to the study being performed in newly diagnosed OSA patients. Low oxygen saturation along with apnea causes numerous inflammatory markers to increase in patients with OSA and characterized systemic inflammatory response. The ongoing inflammatory response plays a role in atherosclerosisis [28]. Based on such data, there was a significant difference between the ODI and apnea-hypopnea index in the OSA patients who did and did not have carotid intima-media hyperplasia in this study. Demİrel et al. [29] put forth in their study that low serum total bilirubin level along with hypertension and hypercholesterolemia is a risk factor for intimal-medial hyperplasia after multiple logistic regression analyses (OR: 1.57, 95\% CI: 1.16-1.93). This study supported the results from the previous studies and total bilirubin levels in patients with OSA were found to be an independent risk factor for carotis intima-media hyperplasia in the multivariate logistic regression analysis. In addition, AHI that shows the severity of OSA was found to be an independent risk factor for carotid intima-media hyperplasia. Thus, OSA-related hypoxia may be indirectly contributed to atherosclerosis progression. Preventing atherosclerosis development is a significant issue. It helps take early protective precautions for diseases that could develop in relation to atherosclerosis. Measuring serum bilirubin levels is a simple procedure. Just as we have done in this study, clarifying the relationship between bilirubin and atherosclerosis in further studies could be improved as it could help determine a preventable risk factor in the development of atherosclerosis. However, more detailed and extensive research is required in order to reveal bilirubin as an independent risk factor for atherosclerosis in a clear manner.

\section{Conclusions}

This study showed low serum total bilirubin levels could be paving the way for the development of subclinical atherosclerosis. This study could hold importance in the sense that it brings people's attention to such an important that is the effect of bilirubin on atherosclerosis. These findings 
have led to the idea that the antioxidant mechanism of bilirubin may play an important role as a protective factor in prone to atherosclerotic conditions such as OSA. Future experiments will help ensure that high levels of bilirubin indicate that the worsening of cIMT in such patients is the "protective guardian" of bilirubin.

\section{Study Limitation}

The limitations of this study could be that this study was cross-sectional and was conducted with a small number of patients; we were not able to investigate the effects of continuous positive airway pressure therapy, which could be a further evidence for the relationship between inflammation and atherosclerosis; markers such as IL-1, IL-6, and TNF- $\alpha$ were not evaluated; atherosclerosis in the patients were examined through non-invasive methods; and coronary angiography was not performed.

\section{$* * *$}

Funding sources: The authors received no financial support for the research, authorship, and/or publication of this article.

Authors' contribution: $\mathrm{HK}$ is responsible for patient selection, analysis, and writing. SÖ contributed to the data collection and editing of the manuscript.

Conflict of interest: The authors declared no potential conflict of interest with respect to the research, authorship, and/or publication of this article.

Data availability: The data sets generated during and/or analyzed during this study are available from the corresponding author on reasonable request.

\section{References}

1. Stec DE, Ishikawa K, Sacerdoti D, Abraham NG: The emerging role of heme oxygenase and its metabolites in the regulation of cardiovascular function. Int J Hypertens 2012, 593-530 (2012)

2. Schwertner HA, Fischer JR: Comparison of various lipid, lipoprotein, and bilirubin combinations as risk factors for predicting coronary artery disease. Atherosclerosis 150, 381-387 (2000)

3. Djousse L, Levy D, Cupples LA, Evans JC, D'Agostino RB, Ellison RC: Total serum bilirubin and risk of cardiovascular disease in the Framingham offspring study. Am J Cardiol 87, 1196-2007 (2001)

4. Novotný L, Vítek L: Inverse relationship between serum bilirubin and atherosclerosis in men: A meta-analysis of published studies. Exp Biol Med 228, 568-571 (2003)

5. Flemons WW: Obstructive sleep apnea. N Engl J Med 347, 498-504 (2002)

6. Mann DL, Zipes DP, Libby P, Bonow RO, Braunwald E (2011): Braunwald's heart disease : A textbook of cardiovascular medicine (9th ed). Elsevier Health Sciences, Philadelphia, PA, p. 897

7. Folsom AR, Kronmal RA, Detrano RC, O'Leary DH, Bild DE Bluemke DA, Budoff MJ, Liu K, Shea S, Szklo M, Tracy RP, Watson KE, Burke GL: Coronary artery calcification compared with carotid intima-media thickness in the prediction of cardiovascular disease incidence: The multi-ethnic study of atherosclerosis (MESA). Arch Intern Med 168, 1333-1339 (2008)

8. Polak JF, Pencina MJ, Pencina KM, O'Donnell CJ, Wolf PA, D'Agostino RB: Carotid-wall intima-media thickness and cardiovascular events. N Engl J Med 36, 5213-221 (2011)

9. Cappelletti A, Astore D, Godino C, Bellini B, Magni V, Mazzavillani M, Pagnesi M, Agricola E, Chiesa R, Colombo A, Margonato A: Relationship between syntax score and prognostic localization of coronary artery lesions with conventional risk factors, plasma profile markers, and carotid atherosclerosis (CAPP Study 2). Int J Cardiol 1, 306-311 (2018)

10. Robertson CM, Gerry F, Fowkes R, Price JF: Carotid intima-media thickness and the prediction of vascular events. Vasc Med 17, 239-248 (2012)

11. Akyuz A, Oran M, Alpsoy S, Mutlu LC, Akkoyun DC, Guzel S, Alp R: Association between serum fetuin-A levels, carotid artery stiffness, and intima-media thickness in patients with normotensive obstructive sleep apnea syndrome. Angiology 65, 607-613 (2014)

12. Mihailovic-Stanojevic N, Miloradović Z, Ivanov M, Bugarski B, Jovović Đ, Karanović D, Vajić UJ, Komes D, Grujić-Milanović J: Upregulation of heme oxygenase-1 in response to wild thyme treatment protects against hypertension and oxidative stress. Oxid Med Cell Longev 2016, 1458793 (2016)

13. Ong KL, Wu BJ, Cheung BMY, Barter PJ, Rye K-A: Association of lower total bilirubin level with statin usage: The United States National Health and Nutrition Examination Survey 1999-2008. Atherosclerosis 219, 728-733 (2011)

14. Wu T, Fung K, Yang C: Unconjugated bilirubin inhibits the oxidation of human low density lipoprotein better than Trolox. Life Sci 54, 477-481 (1994)

15. Mazzone G, Rigato I, Ostrow J, Tiribelli C: Bilirubin effect on endothelial adhesion molecules expression is mediated by the NF$\kappa \mathrm{B}$ signaling pathway. Biosci Trends 3, 151-157 (2009)

16. Wu T-W, Fung KP, Wu J, Yang C-C, Weisel RD: Antioxidation of human low density lipoprotein by unconjugated and conjugated bilirubins. Biochem Pharmacol 51, 859-862 (1996)

17. Wei M, Schwertner H, Zeng QT, Blair SN (2000): Fasting serum bilirubin concentrations and the risk of subsequent coronary heart disease death in men. W. B. Saunders CO., Philadelphia, 32, 314A

18. Vítek L, Jirsa M, Brodanová M, Kalab M, Marecek Z, Danzig V, Novotný L, Kotal P: Gilbert syndrome and ischemic heart disease: A protective effect of elevated bilirubin levels. Atherosclerosis 160, 449-456 (2002)

19. Faizal P, Satheesan B, Vinod B, Thomas Augusti K: Evaluation of antioxidant, lipid peroxidation and toxic effects after pomegranate intake in healthy human volunteers. Int J Clin Med 8, 12-20 (2017)

20. Kundur AR, Santhakumar A., Bulmer AC, Singh I: Mildly elevated unconjugated bilirubin is associated with reduced platelet activation related thrombogenesis and inflammation in Gilbert's syndrome. Platelets 28, 779-785 (2017)

21. Schwertner HA, Jackson WG, Tolan G: Association of low serum concentration of bilirubin with increased risk of coronary artery disease. Clin Chem 40, 18-23 (1994)

22. Hopkins $\mathrm{P}, \mathrm{Wu} \mathrm{L}$, Hunt $\mathrm{S}$, James B: Higher serum bilirubin is associated with decreased risk for early familial coronary artery disease. Arterioscler Thromb Vasc Biol 16, 250-255 (1996)

23. Azen S, Qian D, Mack W, Sevanian A, Selzer R: Effect of supplementary antioxidant vitamin intake on carotid arterial wall intimamedia thickness in a controlled clinical trial of cholesterol lowering. Circulation 94, 2369-2372 (1996)

24. Akboğa MK, Canpolat U, Sahinarslan A, Alsancak Y, Nurkoc S, Aras D, Aydogdu S, Abaci A: Association of serum total bilirubin level with severity of coronary atherosclerosis is linked to systemic inflammation. Atherosclerosis 240, 110-114 (2015) 
25. Huang F-Y, Peng Y, Huang B-T, Yang Y, Pu XB, Chen SJ, Gui YY, Xia TL, Chen F, Liu RS, Zhu Y, Chen M: The correlation between serum total bilirubin and outcomes in patients with different subtypes of coronary artery disease. Clinica Chimica Acta 465, 101-105 (2017)

26. Breimer L, Wannamethee G, Ebrahim S: Serum bilirubin and risk of ischemic heart disease in middle-aged British men. Clin Chem 41, 1504-1508 (1995)

27. Troughton J, Woodside J, Young I, Arveiler D, Amouyel P, Ferrières J, Ducimetière $\mathrm{P}$, Patterson CC, Kee F, Yarnell JW, Evans A: Bilirubin and coronary heart disease risk in the Prospective
Epidemiological Study of Myocardial Infarction (PRIME). Eur J Cardiovasc Prev Rehabil 14, 79-84 (2007)

28. Ciccone MM, Scicchitano P, Zito A, Cortese F, Boninfante B, Falcone VA, Quaranta VN, Ventura VA, Zucano A, Di Serio F, Damiani MF, Resta O: Correlation between inflammatory markers of atherosclerosis and carotid intima-media thickness in obstructive sleep apnea. Molecules 19, 1651-1662 (2014)

29. Demİrel Y, Taş A, Taş F, Bolayir E, Topaktaş S: Evaluation of the effects of serum bilirubin levels on intimal medial hyperplasia of carotis vessels. Erciyes Med J 27, 53-57 (2005) 